\title{
CRÈME: The 2011 Revision of the Cosmic Ray Effects on Micro-Electronics Code
}

J. H. Adams, Jr., A. F. Barghouty, M. H. Mendenhall, R. A. Reed, B. D. Sierawski, J. W. Watts, Jr., and R. A. Weller

\begin{abstract}
:
We describe a tool suite, CRÈME, which combines existing capabilities of CREME96 and CREME86 with new radiation environment models and new Monte Carlo computational capabilities for single event effects and total ionizing dose.
\end{abstract}

Presenting Author:

Robert A. Weller, Vanderbilt University, Department of Electrical Engineering and Computer Science, Nashville, TN. Email: robert.a.weller@vanderbilt.edu. Phone: (615) 343-6027

Contributing Authors:

James H. Adams, Jr., The University of Alabama, Huntsville, AL

Abdulnasser F. Barghouty, NASA Marshall Space Flight Center, Hunstville, AL

Marcus H. Mendenhall, Vanderbilt University, Department of Electrical Engineering and Computer Science, Nashville, TN

Robert A. Reed, Vanderbilt University, Department of Electrical Engineering and Computer Science, Nashville, TN

Brian D. Sierawski, Vanderbilt University, Institute for Space and Defense Electronics, Nashville, TN John W. Watts, Jr., Independent Consultant, Huntsville, AL

Session Preference: Environments

Presentation Preference: Poster

Acknowledgements:

This work was supported by the Autonomous Systems \& Avionic Project of NASA's ETDD Program. Additional support has been provided by the Defense Threat Reduction Agency Basic Research Program and the NASA Electronic Parts and Packaging Program. 


\section{Introduction}

The Cosmic Ray Effects on Microelectronics Code (CRÈME) was first released in 1986 [1]. Over the following ten years there were many advances in our understanding of the space radiation environment.

During this period it was realized that galactic cosmic ray modulation had not only an eleven year cycle but also a twenty-two year cycle [2]. Furthermore it was recognized that the sunspot number is a leading indicator for galactic cosmic ray modulation and could be used to accurately model modulation [3]. Small ${ }^{3}$ He-rich events that were found to be enriched in heavy ions [4], accounting for much of the dispersion in elemental composition reported in CRÈME 86. It was discovered that solar energetic particles (SEPs) were accelerated in coronal mass ejections (CMEs) [5] and this was the source of the largest solar particle events (SPEs) [6]. New data [7],[8] made it possible to model the charge states of solar energetic particles. Also it was shown that rise in the single event upset (SEU) cross section could be described by an integral Weibull distribution [9]. All these and other revisions were included in CRÈME 96 [10].

A decade after the release of CRÈME96 it was becoming obvious that two segments of the site needed to be updated, selected environment models and rate prediction approaches. In this work, we describe the evaluation of environment models and their incorporation into the updated CRÈME web site. We used a chi-squared approach to evaluate three Galactic Cosmic Ray models (a fourth model will be evaluated in the final paper) and selected a single model for the new CRÈME site. We also developed a new model that describes the albedo neutron environment near the surface of the moon. Finally, for environments, this revision also includes a revised description of the transport of the space radiation environment through shielding.

These issues prompted the revision of CREME96 that is described here.. The latest version of CRÈME, together with the two earlier versions, is now available at https://creme.isde.vanderbilt.edu/.

\section{Environmental Models}

II.a. Galactic Cosmic Ray Model: CREME96 used the Nymmik Model [3], which was adopted as a standard by the International Standards Organization (ISO). To choose a model for this revision, we reviewed the models that are available in the literature and chose those that modeled the differential energy spectra over the energy range from at least $10 \mathrm{MeV} / \mathrm{u}$ to $100 \mathrm{GeV} / \mathrm{u}$. The models satisfying these requirements are the revised Nymmik Model [12], the CHIME Model [13], and the Badhwar-O’Neill Model [14].

We evaluated the Badhwar-O’Neill, CHIME and updated Nymmik models by comparing them with measured elemental spectra from an evaluated database we have developed. This database will contain more than 380 measurements of the elemental spectra of GCRs ranging from hydrogen to iron and from $10 \mathrm{MeV} / \mathrm{u}$ to $1 \mathrm{TeV} / \mathrm{u}$. These measurements cover the time period from 1960 to the present. We have compared these individual evaluated spectra with each model by calculating the reduced chi-squared between each model and each spectrum, where reduced chi-squared is defined as:

$$
\left.\frac{\chi^{2}}{v}=\frac{1}{v} \sum_{i=1}^{N} \frac{1}{\sigma_{i}^{2}}\left[\phi_{i}-f\left(E_{i}\right)\right]^{2}\right]
$$


Where $\phi$ is the measured flux at $E_{i}, \sigma_{i}$ is the error in this measurement and $f\left(E_{i}\right)$ is the flux calculated at $E_{i}$ from the model under test. $v$ is $N-p-1$ where $N$ is the number of data points in the spectrum, $p$ is the number of free parameters in the model under test and $\chi^{2} / v$ is the reduced chisquared. $\sigma_{i}$ is proportional to $\sqrt{\square}$ where $n_{i}$ is the number of detected cosmic rays used to construct $\phi_{i}$. Here we have bounded $\sigma_{i}$ to be $\geq 0.1 \phi_{i}$ to account for systematic errors, especially in the determination of the geometrical factor of the instrument. The results are given in Table 1 below. Based on these results we chose the Nymmik model for the revision. Since this evaluation was made, the Badhwar-O'Neill model has itself been revised [15]. We will evaluate this new model and report the results in the full paper to follow this summary.

Table 1: The weighted mean results on the reduced chi-squared for all the spectral fits. The CRÈME 2009 Nymmik Model gives the best fits overall.

\begin{tabular}{|l|c|c|c|}
\hline Model Name & Badhwar-O’Neill & Nymmik & CHIME \\
\hline Hydrogen & 3.45 & 1.28 & 1.37 \\
\hline Helium & 2.21 & 1.38 & 1.69 \\
\hline Oxygen & 0.71 & 1.21 & 0.65 \\
\hline Iron & 1.32 & 1.13 & 1.32 \\
\hline Overall & 2.46 & 1.31 & 1.46 \\
\hline
\end{tabular}

The CRÈME 2009 Nymmik model has an added advantage over other models. Because it uses the sunspot number as a proxy for modulation, it has some ability to predict the level of modulation up to a year in advance. This requires current data in the monthly smoothed sunspot number. These data are obtained periodically from the Solar Influences Data Center at the Royal Belgian Observatory (http://sidc.be/sunspot-data/). See Figure 1 for a comparison with CREME96.

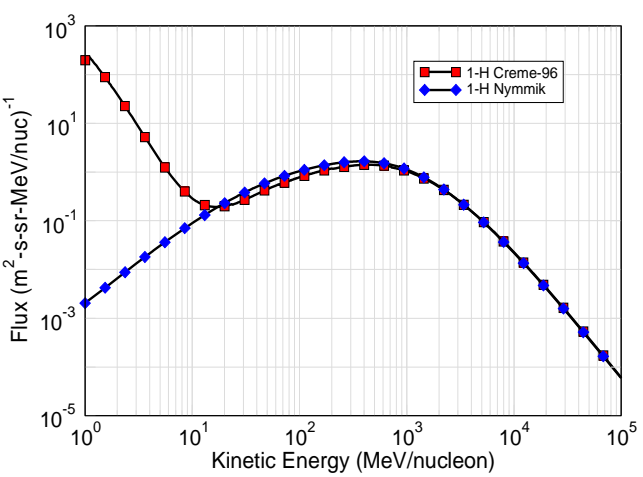

Figure 1. A comparison of the proton flux predicted by CREME96 and CRÈME 2009. Note the large difference for protons below $10 \mathrm{MeV}$, but the general agreement above $10 \mathrm{MeV}$.
II.b. Lunar Neutron Albedo: We have added to CRÈME a model for the neutron albedo coming from the lunar surface due to cosmic rays bombarding the surface. This model is based on a GEANT4 calculation of the albedo from protons and helium nuclei striking the lunar surface. The GEANT4 neutron albedo flux calculations were conducted at discrete, logarithmically-spaced energies over the full energy range of the cosmic ray spectrum. The neutron albedo flux was then weighted to match the proton and helium spectra at the Moon calculated by CRÈME. The results of these calculations are compared with the neutron albedo measured on the Lunar Prospector mission in Figure 2.

\section{New Computational Capabilities}

III.b. HZETRN 1995/NUCFRG2: We revisited the radiation transport code in CRÈME 96 [10] that is used to determine the radiation environment at the location of the part within the spacecraft for a given radiation environment at the surface of the spacecraft. CREME96 used a radiation transport code called UPROP [16] that was developed for transport of cosmic rays 
through cosmic ray detectors and across interstellar space from the cosmic ray sources to Earth. UPROP was chosen because it solves the Boltzmann transport equation using a marching procedure and therefore is much faster than Monte Carlo codes. We compared [17] UPROP with HZETRN (the 1995 version) [18] and two Monte Carlo codes, FLUKA [19] and GEANT4 [20]. Our version of HZETRN was obtained from the NASA COSMIC software collection (currently at http://www.openchannelfoundation.org/cosmic/) in the late 1990s.

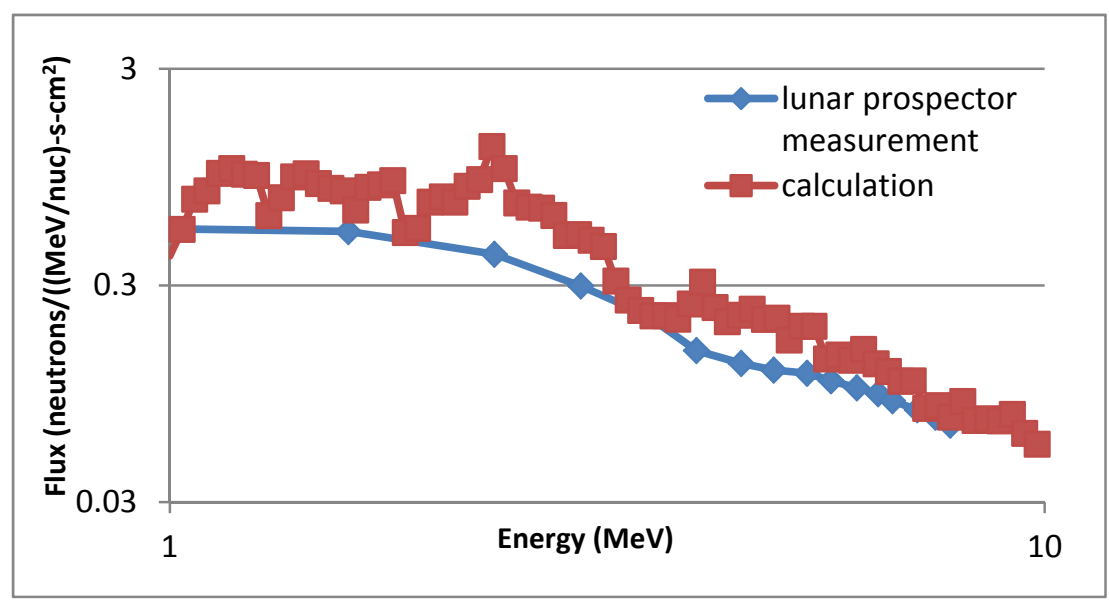

Figure 2: A comparison of the calculated lunar neutron albedo spectrum with spectrum measured on the Lunar Prospector Mission.
HZETRN is a Boltzmann solver that also uses a marching procedure. Our results show that UPROP performed poorer than the other codes for protons and also for heavy ions at low energies. Unlike HZETRN, UPROP does not transport neutrons. This is a deficiency for evaluating radiation effects on the Moon or inside a massive spacecraft like the ISS. We found that HZETRN agreed reasonably well with the Monte Carlo codes except for neutrons at low energies.

As a result of these comparisons we have chosen to include the 1995 version of HZETRN [17] as a users selectable option in the latest revision of CRÈME.

\section{Conclusions}

Revised models for the CRÈME web site that provide improved fidelity and performance are described. The resulting web-based tool combines existing capabilities of CREME96 and CREME86 along with new Monte Carlo computational capabilities to support improved single event effects and total ionizing dose simulations.

\section{References:}

[1] “Cosmic Ray Effects on Microelectronics, Part IV”, James H. Adams, Jr., NRL Memorandum Report 5901, December 31, 1986 (available at www.dtic.mil/cgi-bin/GetTRDoc?AD=ADA176611)

[2] "Characteristics of the 22-Year Modulation of Cosmic Rays as Seen by Neutron Monitors", W. R. Webber and J. A. Lockwood, JGR, 98, 8735-8740, (1988)

[3] “A Model of Galactic Cosmic Ray Fluxes”, R.A. Nymmik, M.I. Panasyuk, T.I. Pervaja, and A.A. Suslov, Nucl. Tracks Radiat. Meas. 20, 427 (1992).

[4] “The heavy-ion compositional signature in He-3-rich solar particle events”, G.M. Mason, D.V. Reames, T.T. von Rosenvinge, B. Klecker and D. Hovestadt, Ap. J., 303, 849-860, (1986).

[5] "A comparison of solar helium-3-rich events with type II bursts and coronal mass ejections", S. Kahler, D.V. Reames, N.R, Sheeley, Jr., R.A. Howard, D.J. Michels and M.J. Koomen, Ap. J., 290, 742-747 (1985).

[6] "Two classes of solar energetic particle events associated with impulsive and long-duration soft Xray flares”, H.V. Cane, R.E. McGuire and T.T. von Rosenvinge, Ap. J., 301, 448-459 (1986). 
[7] "Measurements of the Ionic Charge States of Solar Energetic Particles using the Geomagnetic Field”, R.A. Leske, J.R. Cummings, R.A. Mewaldt, and E.C. Stone, Ap.J. Letters, 452, L149-L152 (1995).

[8] "Solar Energetic Fe Charge State Measurements: Implications for Acceleration by Coronal Mass Ejection-Driven Shocks”, P.R. Boberg, A.J. Tylka, and J.H. Adams, Jr., Ap.J. Letters, 471, L65-L68 (1996).

[9] “Rate Prediction for Single Event Effects - A Critique”, E.L. Petersen, J.C. Pickel, J.H. Adams, Jr. and E.C. Smith, IEEE TNS, 39, 1577-1599 (1992).

[10] "CREME96: A Revision of the Cosmic Ray Effects on Micro-Electronics Code”, Allan J. Tylka, James H. Adams, Jr., Paul R. Boberg, Buddy Brownstein, William F. Dietrich, Erwin O. Flueckiger, Edward L. Petersen, Margaret A. Shea, Don F. Smart, and Edward C. Smith, IEEE TNS, 44, 21502160 (1997).

[11] “Cosmic-Ray-Induced Errors in MOS Devices” James C. Pickel and James T. Blandford, Jr., IEEE TNS, NS-27, 1006-1014 (1980).

[12] "Space environment (natural and artificial) — Galactic cosmic ray model”, R.A. Nymmik, Intl. Stds. Org., ISO 15390, (2004).

[13] "The CRRES/SPACERAD heavy ion model of the environment (CHIME) for cosmic ray and solar particle effects on electronic and biological systems in space”, D.L. Chenette, J. Chen, E. Clayton, T.G. Guzik, J.P. Wefel, M. Garcia-Munoz, C. Lopate, K.R. Pyle, K.P. Ray, E.G. Mullen, D.A. Hardy, IEEE TNS., 41, 2332-2339 (1994).

[14] "Badhwar-O'Neill galactic cosmic ray model update based on advanced composition explorer (ACE) energy spectra from 1997 to present”, P.M., O’Neill, Adv. in Space Res., 37, 1727-1733 (2006).

[15] “Badhwar-O'Neill 2010 Galactic Cosmic Ray Flux Model—Revised” P.M. O'Neill, 57, 3148 - 3153 (2010).

[16] "UPROP A Heavy-ion Propagation Code”, Severn Communications Corporation, SCC Report, 89102, 1989.

[17] "Comparisons of Several Transport Models in Their Predictions in Typical Space Radiation Environments”, Z.W. Lin, J.H. Adams, A.F. Barghouty, S.D. Randeniya, R.K. Tripathi, J.W. Watts and P.P. Yepes, Adv. Sp. Res., 49, 797-806 (2012).

[18] "HZETRN: description of a free-space ion and nucleon transport and shielding computer program", J.W. Wilson, F.F. Badavi, F.A. Cucinotta, J.L. Shinn, G.D. Badhwar, R. Silberberg, C.H. Tsao, L.W. Townsend and R.K. Tripathi, NASA Technical Paper 3495 (1995).

[19] "FLUKA code: description and benchmarking”, G. Battistoni, S. Muraro, P.R. Sala, F. Cerutti, A. Ferrari, S. Roesler, A. Fassò and J. Ranft, The. Proceedings of the Hadronic Shower Simulation Workshop 2006, Fermilab, 6-8 September 2006, M. Albrow, R. Raja eds., AIP Conference Proceeding 896, 31-49 (2007).

[20] “GEANT4 - a simulation toolkit”, S. Agostinelli, J. Allison, K. Amako, J. Apostolakis, H. Araujo, P. Arce, M. Asai, D. Axen, S. Banerjee and D. Zschiesche,. Nucl. Instr. Meth. A506, 250-303 (2003).

[21] “The Trapped Proton Environment in Medium Earth Orbit (MEO)”, G.P. Ginet, S.L. Huston, C.J. Roth, T.P. O'Brien and T.B. Guild, IEEE TNS, 57, 3135-3142 (2010).

[22] "A geomagnetic cutoff rigidity interpolation tool: Accuracy verification and application to space weather”, D.F. Smart, M.A. Shea, A.J. Tylka and P.R. Boberg, Adv. in Sp. Res. 37, 1206-1217 (2006). 